\title{
Confinement of discrete breathers in inhomogeneously profiled nonlinear chains
}

\author{
I. Bena, ${ }^{1}$ A. Saxena, ${ }^{1,2}$ G. P. Tsironis, ${ }^{3}$ M. Ibañes, ${ }^{1}$ and J. M. Sancho ${ }^{1}$ \\ ${ }^{1}$ Department d'Estructura i Constituents de la Matèria, Universitat de Barcelona, 08028 Barcelona, Spain \\ ${ }^{2}$ Theoretical Division, Los Alamos National Laboratory, Los Alamos, New Mexico 87545 \\ ${ }^{3}$ Physics Department, University of Crete and Foundation for Research and Technology-Hellas, P.O. Box 2208, \\ 71003 Heraklion, Crete, Greece
}

(Received 4 October 2002; revised manuscript received 11 December 2002; published 31 March 2003)

\begin{abstract}
We investigate numerically the scattering of a moving discrete breather on a pair of junctions in a FermiPasta-Ulam chain. These junctions delimit an extended region with different masses of the particles. We consider (i) a rectangular trap, (ii) a wedge shaped trap, and (iii) a smoothly varying convex or concave mass profile. All three cases lead to DB confinement, with the ease of trapping depending on the profile of the trap. We also study the collision and trapping of two DBs within the profile as a function of trap width, shape, and approach time at the two junctions. The latter controls whether one or both DBs are trapped.
\end{abstract}

DOI: 10.1103/PhysRevE.67.037601

Introduction. Moving discrete breathers (DBs) in homogeneous nonlinear chains have been studied extensively in recent years [1-3]. The scattering of a DB by isolated impurities has also been explored [4]. If the spatial extent of a DB is about ten or more lattice sites, it can readily be described as an envelope soliton [5]. Therefore, many of the results obtained for solitons and, in particular, those obtained using collective-coordinate approaches in continuum (or, more refined, quasicontinuum [6]) models are directly applicable to DBs' behavior too.

The influence of lattice inhomogeneities of various kinds (mass or interaction parameters) has been considered previously, e.g., in the context of propagation of energy packets along diatomic Toda chains [7], soliton interaction in the Ablowitz-Ladik model [8], soliton propagation in discrete sine-Gordon chain superlattices and aperiodic structures [9], nonlinear diatomic chains [10-12], scattering of Toda solitons at a mass interface [13], etc. Particular attention was paid to the trapping of DBs by an extended impurity region in a nonlinear optical chain with Morse-type on-site potential, intended to model the mechanism of DNA denaturation [14]. However, these latter studies also suggested that many of the features of DBs behavior (e.g., DB trapping, DB interactions, phonon production, DB splitting) are not properly described (qualitatively or quantitatively) within the framework of (quasi)continuum approximations. Valuable information on the effect of discreteness can be obtained from the numerical simulations, and this is the approach we adopt here.

The important issue of the scattering of a DB in the presence of "engineered disorder" has only recently been addressed in the context of an acoustic Fermi-Pasta-Ulam (FPU) chain with a junction [15]. We consider here different kinds of ordered inhomogeneities (as various mass profiles) in FPU chains, obtained by juxtaposing, at a certain distance, two essentially abrupt mass junctions of different types (i.e., heavy-light and light-heavy) thus producing traps with different profiles (insets in Fig. 1). We discuss DB reflection, transmission, trapping, splitting, and confinement phenomena, most of them intimately connected with the discreteness of the chain (as noted above) and the characteristics of the DBs, and therefore difficult to study analytically. Our results
PACS number(s): 05.45.Yv, 63.20.Pw, 63.20.Ry, 87.10.+e

have important implications for realistic situations such as junctions between different electron-phonon coupled chains [16], optical fibers with variable refractive indices [17], and presumably also for energy transport in biomolecules consisting of various functional moieties [18].

Model. The FPU model represents a one-dimensional chain of particles with no on-site potential, with the Hamiltonian for an inhomogeneous chain

$$
H=\sum_{n}\left[\frac{m_{n} \dot{x}_{n}^{2}}{2}+\frac{\alpha}{2}\left(x_{n+1}-x_{n}\right)^{2}+\frac{\beta}{4}\left(x_{n+1}-x_{n}\right)^{4}\right],
$$

where $\alpha$ and $\beta$ denote, respectively, the strengths of the linear and nonlinear nearest-neighbor interactions; $x_{n}$ is the elongation at the $n$th particle (with respect to its equilibrium position), and $m_{n}$ is the mass of the $n$th particle. For simplicity, all these quantities are expressed in dimensionless units. The FPU lattice admits DB-like solutions [19] with periods $T_{D B}$ that are smaller than the minimum period of the phonon spectrum. The details of the model, numerics and how to create various mobile DBs (using the algebraic method [20]) have been given in Ref. [15]. All the results we present below are obtained for a "sandwich" $A-B-A$ structure, in which we fix the parameters of the $A$ part $\left(\alpha_{A}=\beta_{A}\right.$ $\left.=m_{A}=1\right)$ and the interaction constants of the $B$ part $\left(\alpha_{B}\right.$ $=\beta_{B}=1$ ), while choosing different mass profiles in region $B$. A DB with (initial) period $T_{D B}=2.1$ (in order to provide a sense to the profile of this DB, we refer to Fig. 1 in Ref. [15]) moves from one of the regions $A$ of the chain towards region $B$. By "DB's position" at a certain moment we mean the site $n$ with maximum absolute value of the relative elongation $\left|x_{n}-x_{n-1}\right|$.

Profiles. In Fig. 1, we show the trapping of a single DB for five different mass profiles in region $B$. The width of the trap profile is chosen as $L+1=41$ particle sites. For the rectangular case there is no focusing and the DB keeps bouncing back and forth between the two walls (i.e., junctions) of the trap. The DB slows down after each successive rebound indicating that eventually (after a very long time) it would get trapped at some lattice site within region $B$. The second and third panels correspond to a smooth, essentially concave mass profile of the type $m_{i}=0.9+\delta \tanh ^{2}(\lambda \mid L / 2$ $-i \mid), \quad i=0,1, \ldots, L$, where $i$ is the label of the $i$ th particle in the trap, and $\delta$ is chosen, depending on $\lambda$, so that the 

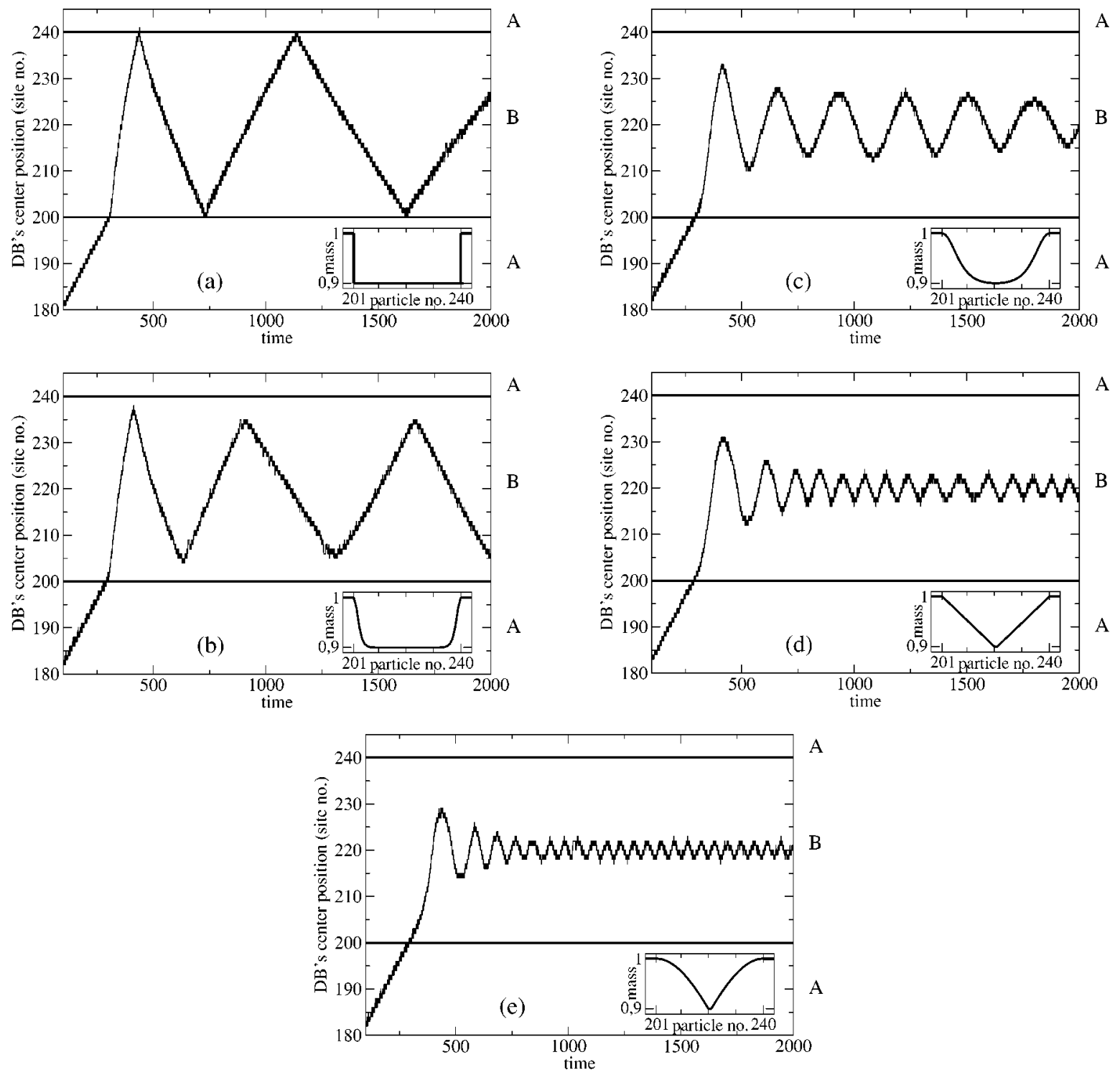

FIG. 1. Trapping of a DB in five different mass profiles (insets) with a trap width of 41 particle sites. The two horizontal lines at site numbers 200 and 240 delimit the trap region. Notice the more efficient focusing as the trap changes from rectangular-passing through smooth concave- to triangular and then to smooth convex. In these and subsequent figures, the quantities plotted are dimensionless.

masses $m_{0}=m_{L}=m_{A}=1$. The values $\lambda=10$ and $\lambda=3$, respectively, allow the concave profile to interpolate between the rectangular trap and a triangular trap (fourth panel). As the profile gets closer to the triangular shape, the DB gets progressively more focused in the center of region $B$. Finally, the last panel shows an essentially convex profile for $\lambda$ $=0.5$; the focusing is even more rapid, as intuitively expected. Recall that a moving DB (that is not an exact solution of the FPU Hamiltonian dynamics) continuously emits phonons, at a very slow rate, while moving through the lattice (due to the discreteness of the lattice). This emission is greatly enhanced inside the profiled trap, leading to DB confinement. Discreteness thus plays an essential role.

Breather collisions. Next, we systematically study the collisions of two DBs as a function of (i) trap width, (ii) DB arrival time, synchronous versus asynchronous, (iii) trap shape, and (iv) whether the two colliding DBs are identical (same frequency and velocity) or different. (i) Figure 2 depicts the effect of trap width for the rectangular case. In the case of synchronous arrival of the two DBs from the opposite sides, for a large trap comprising 41 lattice sites (top panel) the DBs continue to collide symmetrically with each other and with the two walls of the trap with a slight reduction in their velocity at each subsequent collision (the two DBs lose energy through phonon radiation). Note that the "effective" breather size for the current choice of parameters is about ten lattice sites. Therefore, if we choose the trap width to be smaller than the breather size then we may expect a qualitatively different collision behavior.

Indeed, the middle panel of Fig. 2 shows that for a trap of five lattice sites the two breathers first coexist in a (colliding) trapped transient state. However, afterwards one of the DBs is expelled from the trap, while the other one remains trapped with oscillatory collisions with the two walls of the trap (akin to the case in the first panel of Fig. 1). After the interaction, the two DBs have (slightly) different frequencies and velocities compared to their initial values: there is an 
exchange of energy between the DBs in the presence of the "perturbation" represented by the trap. A similar effect, difficult to account for in a collective coordinate description, was reported by Ting and Peyrard [14]. However, we do not find a general tendency toward energy accumulation in the trap. In some cases the DB that "escapes" is more energetic compared to the one arriving at the trap-as in the particular example below. The width of the trap seems to play a role: the larger traps are apparently more efficient in accumulating energy than the smaller ones. For a very narrow trap comprising only two lattice sites (bottom panels of Fig. 2), the behavior depends on the time of arrival. If the DBs arrive simultaneously at the trap, they collide elastically and are reflected back to their respective $A$ sides (no trapping). On the contrary, if one DB arrives slightly before the other one (asynchronous arrival), it gets trapped. When the second DB arrives, it is reflected with some change in frequency and velocity.

(ii) In Fig. 2(a), we showed the case of synchronous arrival of two DBs. The asynchronous arrival in a large rectangular trap is explored in Fig. 3, where we present breather collisions for two different arrival times. If the DBs collide first time at the trap boundary (top panel) then the delayed DB is reflected (usually losing some energy). If they collide asymmetrically inside the trap, then, after a few collisions with the trap boundaries and each other, one of the DBs is absorbed. The heavier resulting DB is finally trapped by the discreteness of the lattice at a site inside the trap. Thus, the collision phenomena are sensitively dependent on arrival time.

(iii) In the previous cases (Figs. 2 and 3), we studied collisions of two DBs in a rectangular trap. To investigate the effect of trap shape on DB collisions, we consider a large triangular trap in Fig. 4 with its width comprising 41 lattice sites, so that the DBs have "enough space" to enter and collide inside the trap. However, due to the focusing effect of such a trap once the DBs are inside it and collide, they are forced to be confined in a progressively smaller space. Eventually they "collapse" and a new DB results. The resultant DB appears to have a larger velocity (the amplitude of oscillation is larger just after the collapse) as if with a tendency to escape the trap. Nevertheless, due to the focusing effect of the trap it is soon confined to the center of the trap. Note that there is always a significant amount of "noise" (phonons and tiny, short lived, breathers resulting from collisions) associated with these collisional events, which seems more pronounced in the case of mass profiles (as compared to our preliminary results for trap profiles in FPU interaction parameters $\alpha$ and $\beta$ ). In Fig. 4, we depicted only the case of synchronous arrival of the two DBs at the triangular trap. If the two DBs arrive asynchronously at the trap (not shown), they may coexist in a transient "bound state" for a short time before collapsing into a single DB.

(iv) We also explored the case of two different DBs (i.e., with different frequencies) arriving at a large trap (41 particle sites). The arrival time is usually asynchronous in this case. As a general rule (results not shown), after entering the trap and colliding (with the walls and with the other, heavier DB), the lighter of the two DBs is "absorbed" by the other one (a
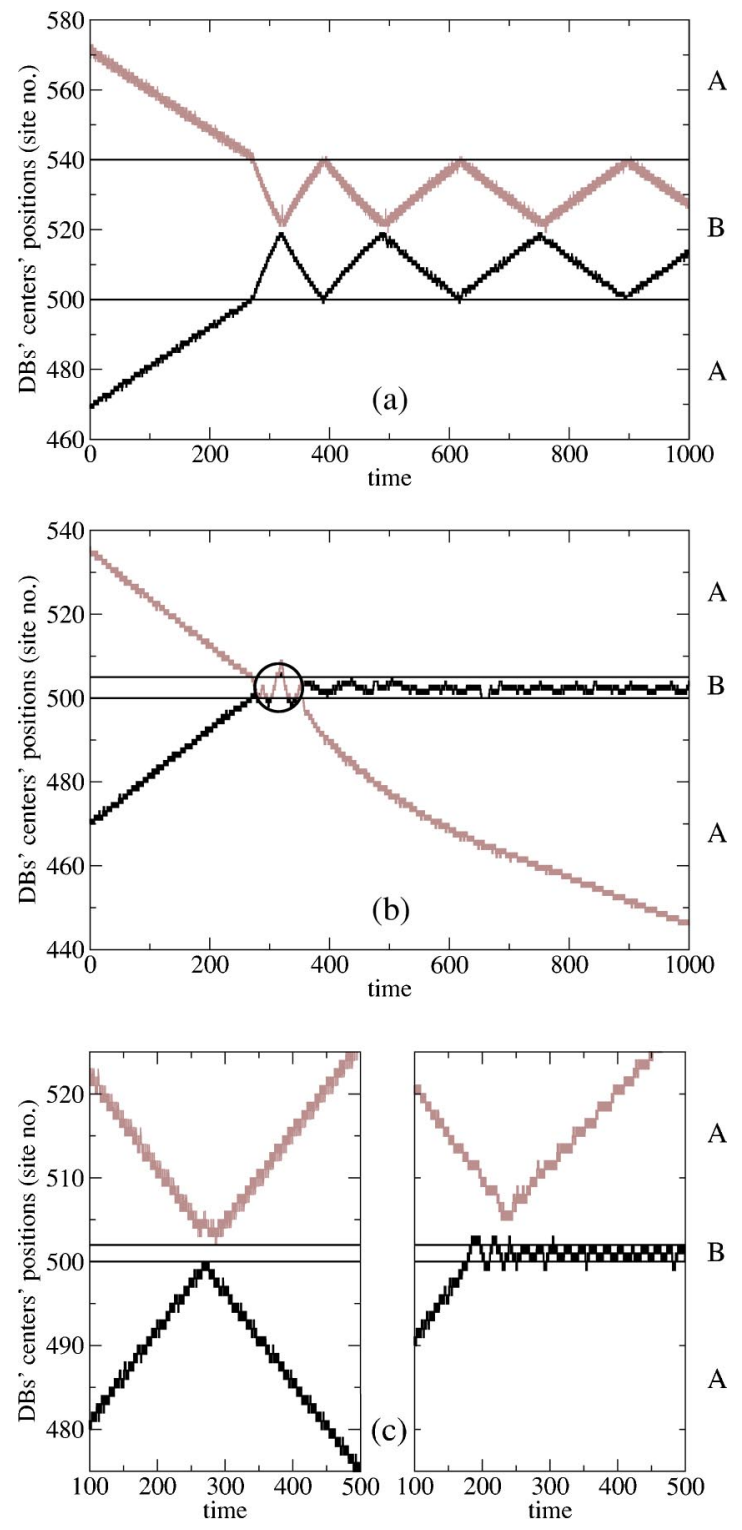

FIG. 2. Scattering of two identical DBs, arriving simultaneously [except for the right panel of Fig. 2(c)] at a rectangular trap from the opposite sides, as a function of trap width. The circle in (b) indicates the coexistence regime of the two DBs.

phenomenon already noticed in different contexts [3]), resulting in a single DB (presumably heavier, i.e., with higher frequency than the colliding ones). The larger the difference in frequency of the two incoming DBs, the quicker the disapperance of the lighter DB. The final, heavier DB is trapped by the discreteness of the lattice (combined, eventually, with the focusing effect of a profiled trap) at some point of the trap, generally its center.

Conclusion. We presented the results of a systematic numerical study of the trapping and collisions of discrete breathers in an inhomogeneous Fermi-Pasta-Ulam chain consisting of mass profiles with various shapes and widths. We also studied the effect of asynchronous arrival time of two DBs at the trap. In all cases quite different DB focusing, merging, and trapping behavior was found. In general, the emerging DBs are different from the initial ones. Qualita- 

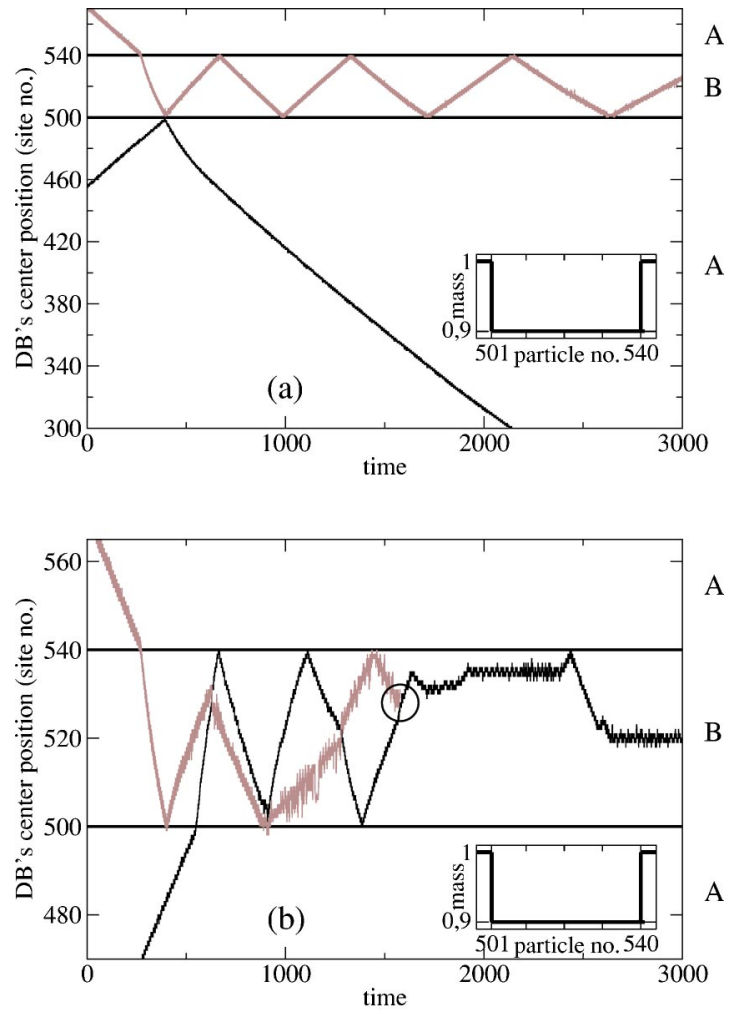

FIG. 3. The collisional behavior of two identical DBs arriving asynchronously at a large rectangular trap depends sensitively on the arrival time [see also Fig. 2(a)]. The circle indicates the "collapse" region where one of the DBs is "absorbed."

tively similar behavior is observed if we study profiles with variable interaction parameters $(\alpha, \beta)$ instead of the mass. Some of the observed DB behavior can be rationalized in terms of estimating the Peierls-Nabarro barrier for inhomo-

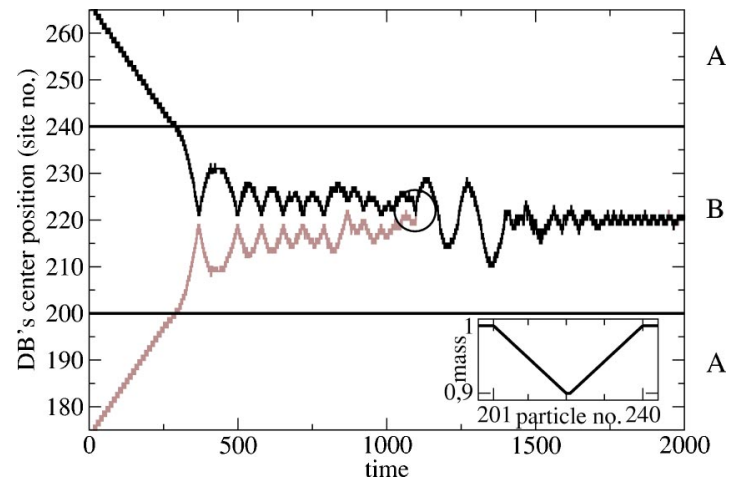

FIG. 4. Collision of two identical DBs arriving at a large triangular trap. The circle indicates the "collapse" region.

geneous chains [15]. Still, a complete quantitative understanding of the cases considered here remains an open question for further study-the use of collective coordinate approach [21] that accounts appropriately for discreteness effects (e.g., the presence of "internal modes" for the FPU breather) is a possibility. We believe the phenomena reported here are qualitatively robust in the sense that (a) the choice of potential (other than FPU) such as, e.g., in the FrenkelKontorova model (see also Ref. [14] for Morse-type potentials) should give similar results and (b) these phenomena persist under (small) perturbations. Finally, we suggest that DBs in electron-phonon coupled chains [16], pulse propagation in optical fibers [17], and energy transport in biomolecules [18] may provide a physical realization of the phenomena found in this study.

We thank A. R. Bishop for fruitful discussions. This work was supported by the European Union under the RTN project LOCNET (Project No. HPRN-CT-1999-00163) and by the U.S. Department of Energy. A.S. gratefully acknowledges financial support from Iberdrola (Spain).
[1] G.P. Tsironis et al., Europhys. Lett. 57, 697 (2002); M. Ibañes et al., Phys. Rev. E 65, 041902 (2002); S.R. Bickham et al., Phys. Rev. B 47, 14206 (1993); S.R. Bickham et al., ibid. 45, 10344 (1992).

[2] S. Flach and K. Kladko, Physica D 127, 61 (1999); S. Aubry and T. Cretegny, ibid. 119, 34 (1998); D. Chen et al., Phys. Rev. Lett. 77, 4776 (1996).

[3] K.Ф. Rasmussen et al., Eur. Phys. J. B 15, 169 (2000); T. Dauxois and M. Peyrard, Phys. Rev. Lett. 70, 3935 (1993).

[4] See, e.g., W. Chen et al., Phys. Rev. B 65, 134302 (2002), and references therein; F. Zhang, Phys. Rev. E 58, 2558 (1998); K. Forinash et al., ibid. 49, 3400 (1994).

[5] For FPU chains, see, e.g., Yu.A. Kosevich and S. Lepri, Phys. Rev. B 61, 299 (2000). Similar envelope-soliton approximations are also valid for DBs in optical chains.

[6] Ch. Claude et al., Phys. Rev. B 47, 14228 (1993); R. Scharf and A.R. Bishop, Phys. Rev. A 43, 6535 (1991).

[7] P.C. Dash and K. Patnaik, Phys. Rev. A 23, 959 (1981); F. Mokross and H. Büttner, ibid. 24, 2826 (1981).

[8] V.V. Konotop et al., Phys. Rev. E 48, 563 (1993).
[9] F. Dominguez-Adame et al., Phys. Rev. E 52, R2183 (1995).

[10] A.V. Zolotaryuk et al., Physica B 296, 251 (2001); R. Livi et al., Nonlinearity 10, 1421 (1997).

[11] A.V. Gorbach et al., Phys. Solid State 43, 2171 (2001).

[12] A. Xu et al., Phys. Rev. B 58, 721 (1998).

[13] T. Klinker and W. Lauterborn, Physica D 8, 249 (1983).

[14] M. Hisakado, Phys. Lett. A 227, 87 (1997); J.J.-L. Ting and M. Peyrard, Phys. Rev. E 53, 1011 (1996); M. Techera et al., Phys. Rev. A 42, 1008 (1990).

[15] I. Bena et al., Phys. Rev. E 66, 036617 (2002).

[16] B.I. Swanson et al., Phys. Rev. Lett. 82, 3288 (1999).

[17] S. Burtsev et al., Phys. Rev. E 52, 4474 (1995); G. P. Agrawal, Nonlinear Fiber Optics (Academic Press, Boston, 1995).

[18] D. Hennig, Phys. Rev. E 64, 041908 (2001).

[19] G. James, C. R. Acad. Sci., Ser. I: Math. 332, 581 (2001); S. Aubry et al., Discrete Contin. Dyn. Syst. Ser. B 1, 271 (2001); J.B. Page, Phys. Rev. B 41, 7835 (1990); A.J. Sievers and S. Takeno, Phys. Rev. Lett. 61, 970 (1988)

[20] G.P. Tsironis, J. Phys. A 35, 951 (2002).

[21] C.R. Willis et al., Phys. Rev. B 33, 1904 (1986). 